\title{
Input to V2 Thin Stripes Arises from V1 Cytochrome Oxidase Patches
}

\author{
Lawrence C. Sincich and Jonathan C. Horton \\ Beckman Vision Center, University of California, San Francisco, San Francisco, California 94143
}

Parallel streams from the primary visual cortex (V1) to the second visual area (V2) are thought to mediate different aspects of visual perception in primates. One hypothesis is that the projection from cytochrome oxidase patches to thin stripes is responsible for color, whereas a separate pathway from interpatches to pale stripes mediates form. Recently, the notion of segregated pathways has been challenged by a report showing that patches and interpatches project equally to thin stripes. We made injections of a retrograde tracer, cholera toxin-B (CTB-Au), into macaque V2 thin stripes and counted the number of labeled cells in patches versus interpatches in layer $2 / 3$. Analysis of eight thin-stripe injections showed that a mean of $81 \%$ of labeled cells were located in patches (defined as $33 \%$ of the surface area of V1). This result confirms that the projection to thin stripes arises predominately from patches. To assess the segregation of patch and interpatch projections, we injected CTB-Au in a pale stripe and horseradish peroxidase in an adjacent thin stripe. In both successful cases, interdigitated fields of labeled cells were present in V1. Less than $1 \%$ of cells were double-labeled, indicating that the populations of cells supplying thin stripes and pale stripes are quite independent. This finding means that different signals are likely conveyed by patches and interpatches to $\mathrm{V} 2$.

Key words: visual cortex; column; cytochrome oxidase; flat mount; color vision; cholera toxin

\section{Introduction}

In primates, the main pathway serving visual perception goes from the retina via the lateral geniculate nucleus to area V1. From there, most signals are conveyed to area V2 before distribution to higher cortical areas. To understand the basic circuitry of vision, it is vital to know how the projections between V1 and V2 are organized. In V1, cytochrome oxidase histochemistry has revealed a regular array of darkly staining regions, known as patches (blobs, puffs) (Horton and Hubel, 1981; Wong-Riley and Carroll, 1984). In V2, the enzyme has shown a repeating pattern of pale-thin-pale-thick stripes (Tootell et al., 1983; Horton, 1984). Livingstone and Hubel (1984) explored the anatomical relationship between the cytochrome oxidase (CO) compartments in V1 and V2. They found a projection arising exclusively from patches in layer $2 / 3$ that terminates in thin stripes. This result was confirmed by Sincich and Horton (2002a), who noted additional sparse projections from patches in layers $4 \mathrm{~A}, 4 \mathrm{~B}$, and $5 / 6$.

The regions between patches, known as interpatches, contain cells that project to pale stripes (Livingstone and Hubel, 1984) and to thick stripes (Sincich and Horton, 2002a). The projection from V1 to V2 is divided, therefore, into two distinct streams:

Received June 4, 2005; revised Sept. 23, 2005; accepted Sept. 23, 2005.

This work was supported by a grant from the Larry L. Hillblom Foundation and by Grants EY10217 (J.C.H.), EY13676 (L.C.S.), and EY02162 (Beckman Vision Center) from the National Eye Institute. The California Regional Primate Research Center is supported by National Institutes of Health Base Grant RR00169. Cristina Jocson helped with cell plotting, Amar Marathe assisted with data analysis, and Robin Troyer provided animal care. We thank David Copenhagen and Erik Ullian for comments on the final manuscript.

Correspondence should be addressed to Dr. Jonathan C. Horton, Beckman Vision Center, University of California,

San Francisco, 10 Koret Way, San Francisco, CA 94143-0730. E-mail: hortonj@vision.ucsf.edu.

DOI:10.1523/JNEUROSCI.3313-05.2005

Copyright $\odot 2005$ Society for Neuroscience $\quad$ 0270-6474/05/2510087-07\$15.00/0 patches $\rightarrow$ thin stripes, interpatches $\rightarrow$ pale and thick stripes. Livingstone and Hubel (1988) have proposed that the pathway from patches to thin stripes mediates color perception, whereas the pathway from interpatches to pale stripes serves form perception. This theory has generated some controversy (for review, see Gegenfurtner and Kiper, 2003). Whether correct or not, one can at least say that the dichotomy between patch and interpatch projections to V2 is likely to segregate one or more cardinal properties related to vision.

Recently, Xiao and Felleman (2004) questioned the existence of a dichotomy in the projections from V1 to V2. After tracer injection into thin stripes, they reported a greater density $(2.8: 1)$ of labeled cells in patches, consistent with previous studies. However, because interpatches have about twice the surface area of patches, they found the same total number of labeled cells in each compartment. This equal patch/interpatch input to thin stripes led Xiao and Felleman (2004) to conclude that their results "call into question the proposition that different $\mathrm{CO}$ compartments in V1 and V2 are connected in parallel to form highly segregated functional streams."

To address these discrepant studies, we performed a quantitative analysis of the projection from V1 by making single-tracer injections into V2 thin stripes. We also made dual-tracer injections into adjacent thin and pale stripes to assess directly the degree of anatomical segregation in the patch and interpatch projections. These new data confirm previous studies reporting a tightly segregated pathway from patches to thin stripes.

\section{Materials and Methods}

Experimental animals. Experiments were conducted in 17 normal adult macaques following procedures approved by the University of California, San Francisco Committee on Animal Research. Anesthesia and sur- 
gery were performed as described previously (Sincich and Horton, 2003). Pressure injections of $140 \mathrm{nl}$ of $0.1 \%$ gold-conjugated cholera toxin-B subunit (CTB-Au; List Biological, Campbell, CA) (Llewellyn-Smith et al., 1990) were made every 5-6 $\mathrm{mm}$ along the posterior lip of the lunate sulcus in V2 at a depth of $1000 \mu \mathrm{m}$. This depth was chosen to target layers 3 and 4, which receive most axon terminals from V1 (Rockland and Pandya, 1979; Lund et al., 1981; Weller and Kaas, 1983; Van Essen et al., 1986; Rockland and Virga, 1990; Sincich and Horton, 2002b). The injections produced a deposit of tracer $\sim 1 \mathrm{~mm}$ in diameter (range, $0.5-1.3$ $\mathrm{mm}$ ), after silver intensification, that usually spanned layers $2-5$. It is difficult to know the uptake zone for actual transport from any tracer injection site, but CTB-Au injections smaller than $\sim 0.4 \mathrm{~mm}$ in diameter failed to produce detectable retrograde labeling in V1. This observation implies that the tracer uptake zones were considerably smaller than the injection sites.

After completing the injections in each hemisphere, the dura was sutured and the bone flap replaced. In some animals, one eye was enucleated in connection with an unrelated experiment. There is no reason to believe that this procedure had any impact on the results presented here. Buprenorphine $(0.02 \mathrm{mg} / \mathrm{kg}$, i.m.) was administered postoperatively every $8 \mathrm{~h}$ until the animal recovered from surgery. After $2-3 \mathrm{~d}$ for transport, the animals were given a lethal dose of pentobarbital $(150 \mathrm{mg} / \mathrm{kg})$ and perfused with $2 \mathrm{~L}$ of $0.9 \%$ saline followed by $1 \mathrm{~L}$ of $1 \%$ paraformaldehyde in $0.1 \mathrm{~m}$ phosphate buffer, $\mathrm{pH}$ 7.4. Flat mounts were prepared containing V1 and V2 (Sincich et al., 2003). They were cut parallel to the pial surface at $50 \mu \mathrm{m}$, dried on slides, and processed for CO (Wong-Riley, 1979). The sections were then double-labeled by silver intensification of the CTB-Au with an IntenSE-M kit (Amersham Biosciences, Little Chalfont, UK).

In some monkeys, paired tracer injections were made of CTB-Au and wheat germ agglutinin conjugated to horseradish peroxidase (WGA$\mathrm{HRP}$ ). Alternate sections were processed either for CO or for CTB-Au and WGA-HRP. Free-floating sections were silver intensified to reveal CTB-Au and then exposed for $5 \mathrm{~min}$ to $2 \%$ sodium thiosulfate. Afterward, they were reacted according to a tetramethylbenzidineammonium molybdate protocol (LeVay and Voigt, 1990), followed by diaminobenzidine stabilization (Rye et al., 1984).

Data analysis. For each analyzed V2 injection, we selected the tissue section containing the densest retrograde labeling in layer 3 of V1. Each CTB-Au-labeled cell was plotted by aid of a camera lucida attachment at $200 \times$ magnification, using crossed Polaroid (Cambridge, MA) filters and dark-field illumination. Its location was entered into a computer using a Cintiq 15x display tablet (Wacom, Vancouver, WA) in conjunction with AutoCAD 2002 software (Autodesk, San Rafael, CA).

To quantify the intensity of CO staining, digital photographs were taken with a Spot RT color CCD camera (Diagnostic Instruments, Sterling Heights, MI) mounted on an Olympus (Melville, NY) SZH10 microscope. Vessel profiles were filled in with pixels of the mean gray level value of the image using Photoshop 7.0 (Adobe Systems, San Jose, CA). To correct for global changes in section density (caused by slight shifts in layer 3 depth), a low-pass Fourier-filtered (cutoff frequency, 1.1 cycles/ $\mathrm{mm}$ ) image was subtracted from the original image using Matlab (MathWorks, Natick, MA). The resulting image was then blurred with a Gaussian filter $(\sigma=45 \mu \mathrm{m})$ and divided into six zones of equal area based on the density of CO staining. The darkest two zones (33\% area) were designated as patches; the palest four zones were defined as interpatches (66\% area). The fraction of V1 assigned to the patch compartment is arbitrary. Typically, most investigators have designated approximately one-third of the striate surface area as patches (Horton, 1984; Purves and LaMantia, 1993; Farias et al., 1997; Sincich and Horton, 2002b). Obviously, if patches are defined as a smaller percentage of $\mathrm{V} 1$, the proportion of cells projecting to thin stripes that arises from patches will fall. Conversely, if patches are defined more generously, the proportion will rise.

Xiao and Felleman (2004) performed a secondary analysis, namely, a measurement of the distance from each labeled cell to its closest patch center. They defined a patch center as "the spot with the densest CO staining within a blob." However, a patch often contains several peaks of maximum density, and these are not always in the center of the patch. In addition, patches are oblong and irregular in shape. Consequently, cells

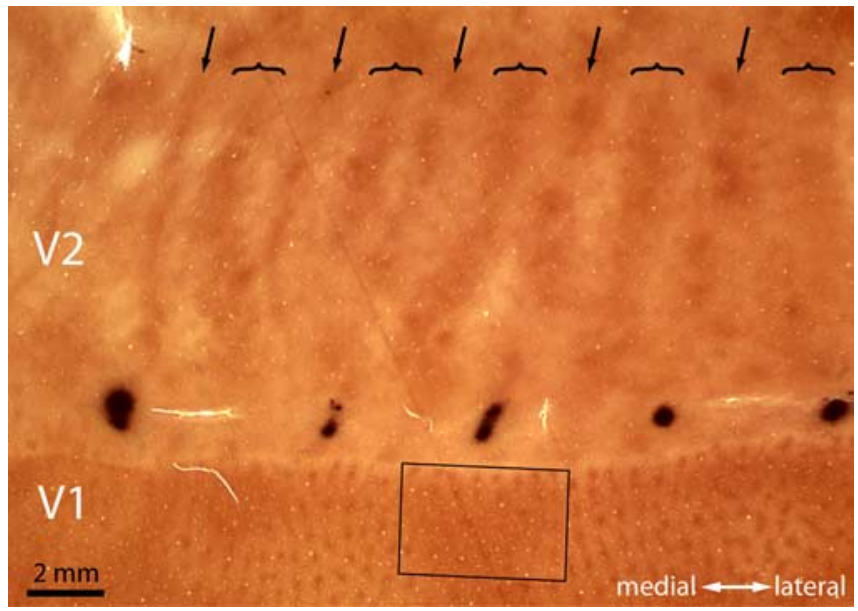

Figure 1. Tracer injection into a thin stripe. This $\mathrm{CO}$ section at the layer $3-4 \mathrm{~A}$ junction contains a string of five CTB-Au injections made along the exposed lip of the right lunate sulcus, near the V1 border. The lunate sulcus has been opened and flattened to show clearly the pattern of V2 stripes (arrow, thin; bracket, thick). The middle injection is situated within a thin stripe. The rectangle corresponds to the field in Figure 2 containing retrogradely filled cells.

outside patches can sometimes be closer to patch centers than cells inside patches. For these reasons, we did not use this analysis.

A two-way $\chi^{2}$ test was used to determine whether the distribution of retrogradely labeled cells in patches and interpatches differed significantly from chance.

\section{Results}

Because tracer injections were made blindly into V2 with respect to stripe type, many were unsuitable for analysis. Of 187 injections, 110 were rejected because they contaminated an adjacent stripe, spread across the border into V1, were too small for transport, or hit an unidentifiable stripe. Only 77 injections landed in a single stripe of clear identity, 17 in thin stripes. Stripe identity was ascertained before silver intensification of the sections and, therefore, before knowing anything about the distribution of labeled cells in V1. This avoided any potential bias in our identification of stripe type.

In a preliminary account, we reported that all 17 thin stripe injections resulted in retrograde labeling of cells located in patches (Sincich and Horton, 2002a). However, we did not plot the location of individual CTB-Au-filled cells. Instead, the distribution of cells was inferred by thresholding the density of silver grains at low power to define contours of label intensity. These contours appeared to encircle the CO patches. Xiao and Felleman (2004) pointed out that this approach could miss a large number of cells scattered at comparatively low density throughout the interpatches. Here, we provide a more rigorous analysis based on a plot of the position of each CTB-Au-filled cell, prepared from 8 of 17 cases. These cases were selected because the CTB-Au injection in V2 was made into the core of a thin stripe, guaranteeing that there was little spillover into an adjacent pale stripe.

Figure 1 shows a CTB-Au injection in a thin stripe, located $\sim 500 \mu \mathrm{m}$ from the $\mathrm{V} 1$ border. Because the retinotopic maps in V1 and V2 are arranged like mirrored images, the injection produced a field of labeled cells just across the border in V1. These cells were distributed into 25-30 clusters, which appeared bright in dark field (Fig. 2a). The density of CO staining in the section was divided into six zones of equal area (Fig. $2 b, c)$. The position of each CTB-Au-filled cell was then superimposed on the contours of CO density (Fig. $2 d$ ). The clusters of retrogradely labeled cells 

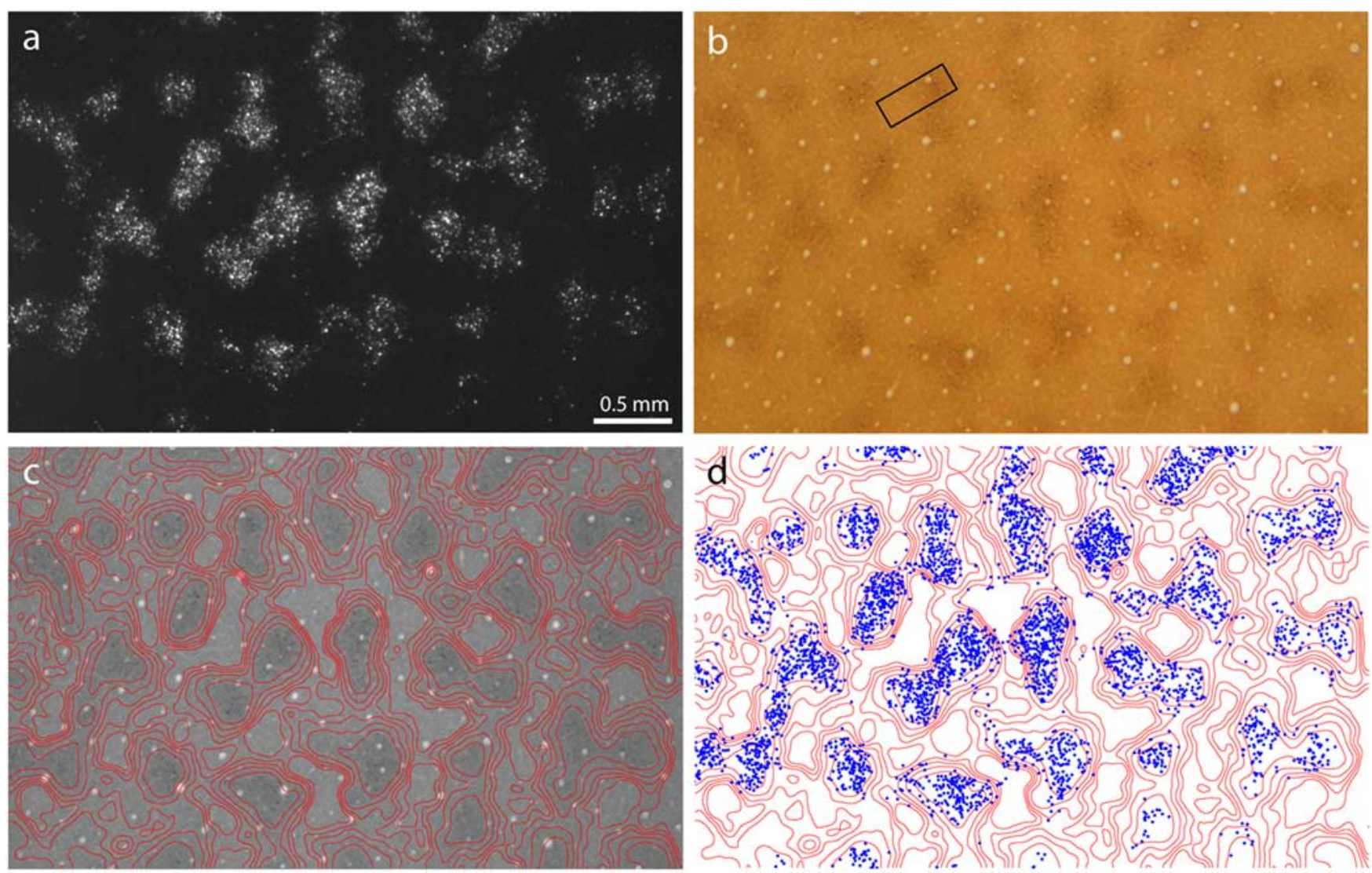

Figure 2. Alignment of CTB-Au-filled cells with CO patches. $\boldsymbol{a}$, Clusters of retrogradely labeled cells are visible in dark-field illumination. The field corresponds to the rectangle in Figure 1 but is from a section $200 \mu \mathrm{m}$ more superficial that passes through the middle of layer 3 . $\boldsymbol{b}$, The same section viewed in bright field, showing the C0 patches. The boxed region is shown in supplemental Figure 88 (available at www.jneurosci.org as supplemental material). $\boldsymbol{c}$, Density contours superimposed on $\boldsymbol{b}$ divide the intensity of $\mathrm{CO}$ activity into six equal zones. The darkest and lightest zones appear greatest in area, but this is an illusion. $\boldsymbol{d}$, A plot of the distribution of (TB-Au-filled cells shows that they are clustered preferentially in CO patches.

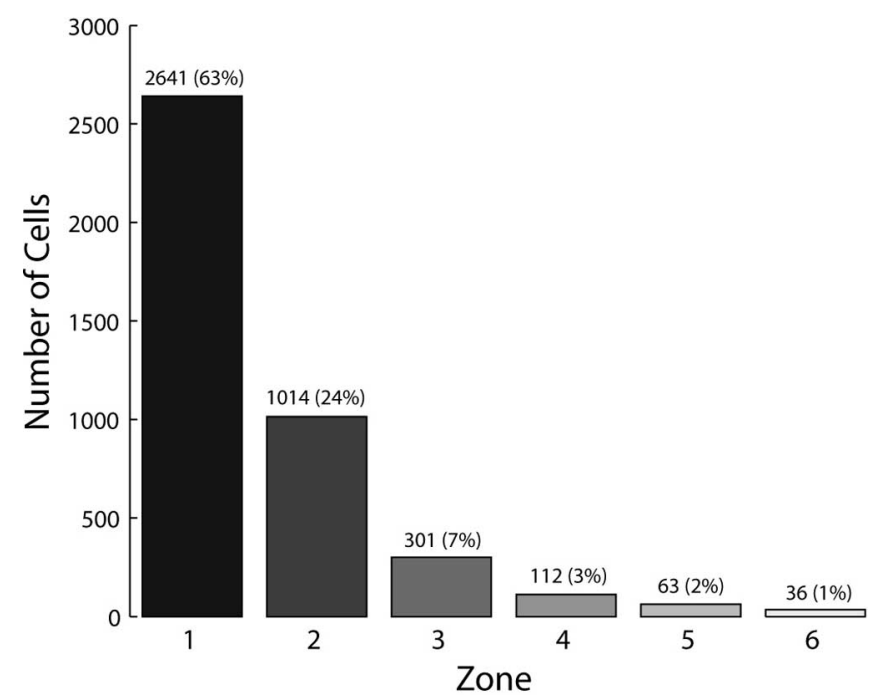

Figure 3. Cell distribution as a function of $\mathrm{CO}$ zone. The histogram shows the distribution of the labeled cells in Figure $2 A$ as a function of $C 0$ density zone. The majority of cells (87\%) are located in zones 1 and 2, which correspond to the $\mathrm{CO}$ patches. These data are from the middle injection in Figure 1 only.

were found to coincide with the CO patches. Large expanses of tissue in the interpatches were devoid of labeled cells.

Figure 3 shows the number of cells in each of the six $\mathrm{CO}$ compartments. Zones 1 and 2, which correspond to the $\mathrm{CO}$ patches, together contained 3655 of the 4167 cells $(87 \%)$ in this tissue section. Only $13 \%$ of cells were located in the interpatches (zones 3-6). In every case, we performed our counts in the most densely labeled section in layer $2 / 3$. We visually inspected all other sections in layer $2 / 3$ and determined that the CTB-Aulabeled cells were concentrated to a similar degree in the patches. Thus, V1 cells projecting to thin stripes are organized into vertical columns, centered within patches.

The location of each CTB-Au-filled cell was plotted in one section from each of the $\mathrm{V} 1$ projection fields for eight thin stripe injections. Altogether, 30,236 cells were plotted using a camera lucida microscope attachment. Figure 4 shows the mean percentage of cells located in each of the six zones for all eight cases (the actual cell counts are provided in supplemental Table 1, available at www.jneurosci.org as supplemental material). The seven remaining cases (injections 2-8), including images of injection sites in V2, are illustrated in supplemental Figures S1-S7 (available at www.jneurosci.org as supplemental material). In total, $81 \%$ of cells were located in patches (zones 1 and 2). A two-way $\chi^{2}$ test revealed that cells were significantly more likely to be located in patches than interpatches $\left(p<10^{-10}\right)$.

The graph in Figure 4 shows that the density of cells projecting to thin stripes fell steadily as CO staining became weaker. Zone 6, located in the middle of interpatches, contained only $2 \%$ of the labeled cells. This result implies strongly that patches and interpatches project preferentially to different stripe types. The most direct way to test this idea is to make dual tracer injections in V2. In 7 of 17 monkeys, we made paired injections of CTB-Au and 


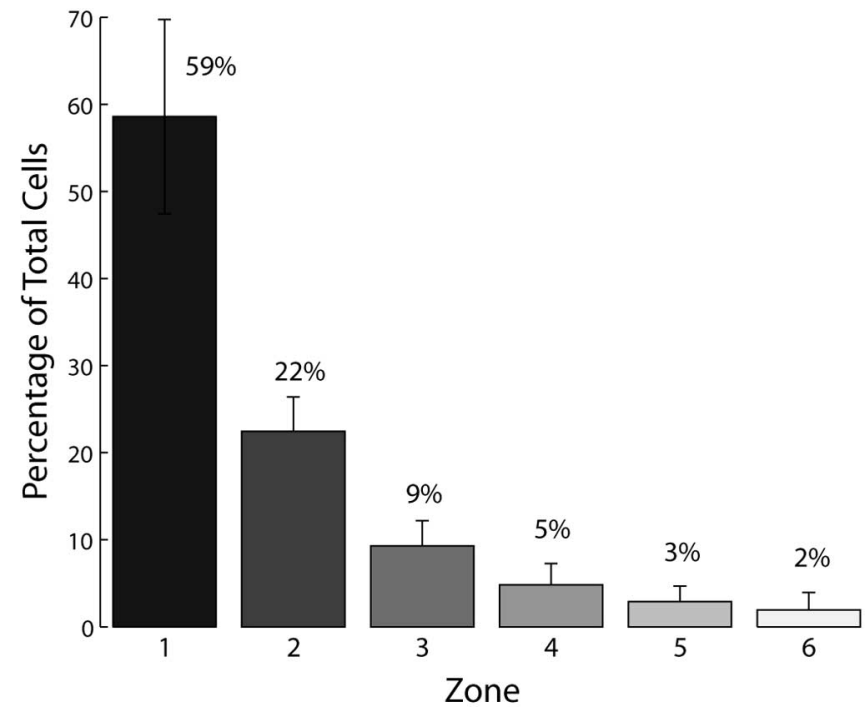

Figure 4. Percentage of cells projecting to thin stripes as a function of $\mathrm{CO}$ density zone. The histogram, combining data from eight injections, shows that $81 \%$ of cells projecting to thin stripes are located in patches (zones 1 and 2). Error bars denote SDs.

WGA-HRP, spaced $1.25 \mathrm{~mm}$ apart. In two cases, the injections landed cleanly in adjacent thin and pale stripes.

Figure 5 illustrates one of the two successful dual-tracer experiments. The CTB-Au injection filled a pale stripe, whereas the WGA-HRP injection hit a thin stripe. The two tracers did not overlap. In V1, interdigitated populations of cells labeled either with CTB-Au or with WGA-HRP were present (Fig. 6a). Comparison with an adjacent section stained for CO (Fig. 6b) showed that the CTB-Au cells $(n=2064)$ filled by the pale-stripe injection were located mostly in interpatches, whereas the WGA-HRP cells $(n=724)$ from the thin-stripe injection were concentrated in patches (Fig. $6 c$ ). Only three cells $(<0.5 \%)$ were doublelabeled. Figure 7 shows histograms of the distribution of labeled cells for each injection. The patches contained $79 \%$ of the WGAHRP-filled cells labeled by the thin-stripe injection. This figure was consistent with the mean of $81 \%$ obtained from the eight examples of single CTB-Au tracer injections. The interpatches contained $89 \%$ of the CTB-filled cells, resulting from the palestripe injection. A similar result was found in the second dualtracer case. The paucity of double-labeled cells was not caused by interference between WGA-HRP and CTB-Au, because doublelabeled cells are abundant after injection of these two tracers into adjacent pale and thick stripes (Sincich and Horton, 2002a).

Although plotting the actual location of each labeled cell sets the highest evidentiary standard, there is an advantage to an analysis based on the threshold of the reaction product density in a field of labeled cells, as performed previously (Sincich and Horton, 2002a). The cells in the middle of a CO patch tend to be more densely labeled than cells at the fringe of a patch (supplemental Fig. S8, available at www.jneurosci.org as supplemental material). This difference in the strength of cell labeling probably correlates with the number of synaptic contacts in V2 at the tracer injection site and is therefore a rough measure of the strength of innervation to a given thin stripe. This useful information is lost when cells are plotted as uniform dots (Fig. 2, compare $a, d$ ), because all cells are weighted equally, regardless of how well they are filled. Therefore, counts of labeled cells underestimate how strongly the projection from V1 to thin stripes is biased in favor of patches. A more accurate, albeit less quantitative, impression is
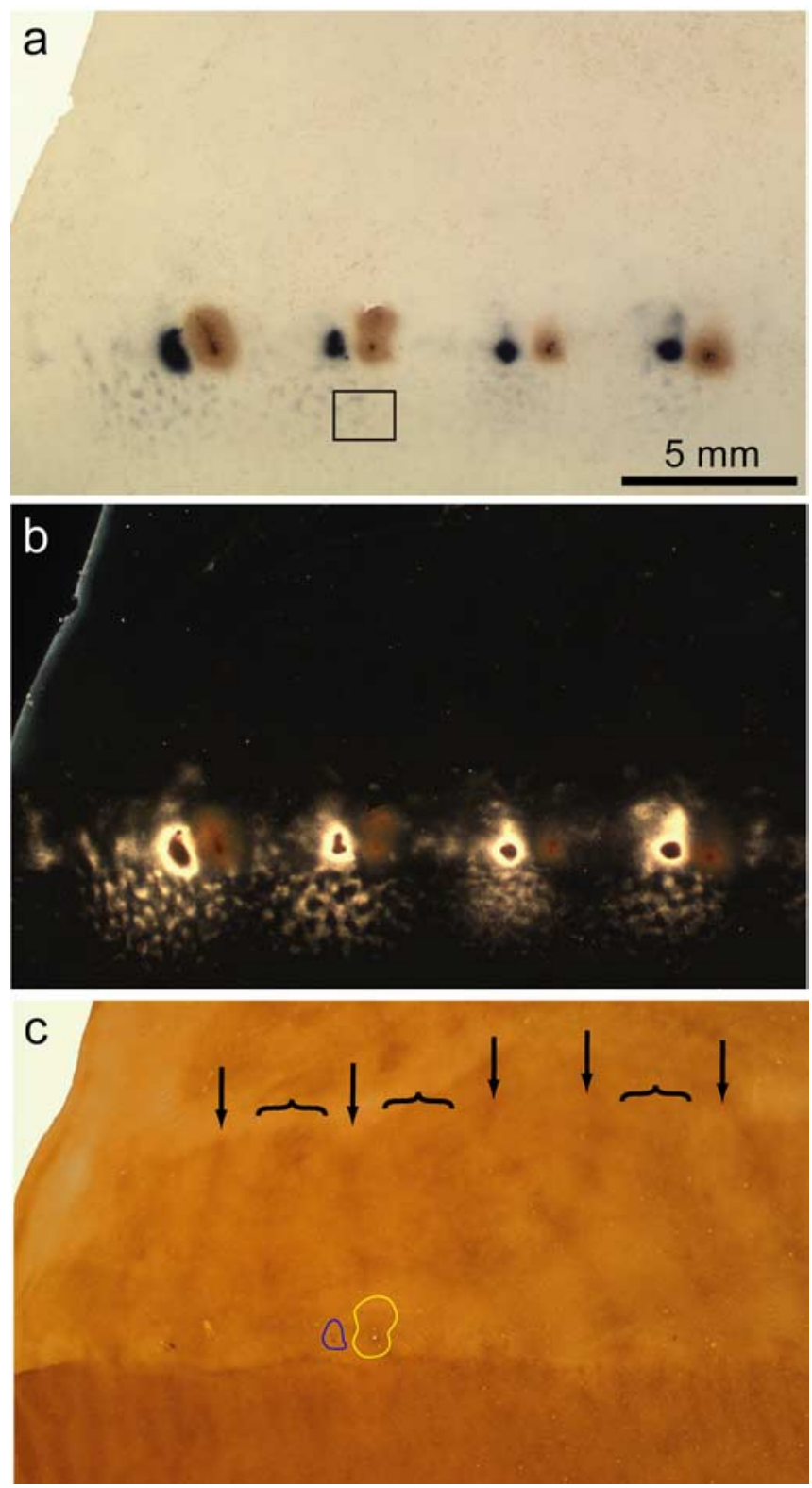

Figure 5. Dual tracer injection into V2. a, Double-labeled section, showing pairs of CTB-Au (black) and WGA-HRP (brown) injections in layer 3 . The second pair from the medial edge of the section landed in an adjacent pale (CTB-Au) stripe and a thin (WGA-HRP) stripe. The boxed region is shown in Figure 6. $\boldsymbol{b}$, The same section, viewed in dark field through crossed Polaroid filters, showing that the two tracer injections do not overlap in V2. A cloud of label from CTBAu-filled cells is visible in V1, with large gaps corresponding to $\mathrm{CO}$ patches. C, $\mathrm{C} 0$ section, 250 $\mu \mathrm{m}$ deeper, showing the pattern of $\mathrm{CO}$ stripes in V2. It was aligned with the more superficial section by comparison of blood vessels and by noting the location of pipette tip artifacts for the WGA-HRP and the purple CTB-Au injection. Outlines of the injection sites in $\boldsymbol{a}$ have been superimposed to facilitate comparison of the tracer deposits and $\mathrm{CO}$ stripes. Arrows, Thin stripes; brackets, thick stripes.

gained simply by looking at low power at the labeling pattern in the microscope (Fig. 2a).

\section{Discussion}

V1 dispenses its output to numerous cortical areas, but V2 receives by far the largest projection [Sincich and Horton (2002b), their Fig. $2 B$ ]. For this reason, an accurate description of the organization of connections between $\mathrm{V} 1$ to $\mathrm{V} 2$ is required to understand how visual signals are transformed by extrastriate cortex. In this report, we show that the input to V2 thin stripes 

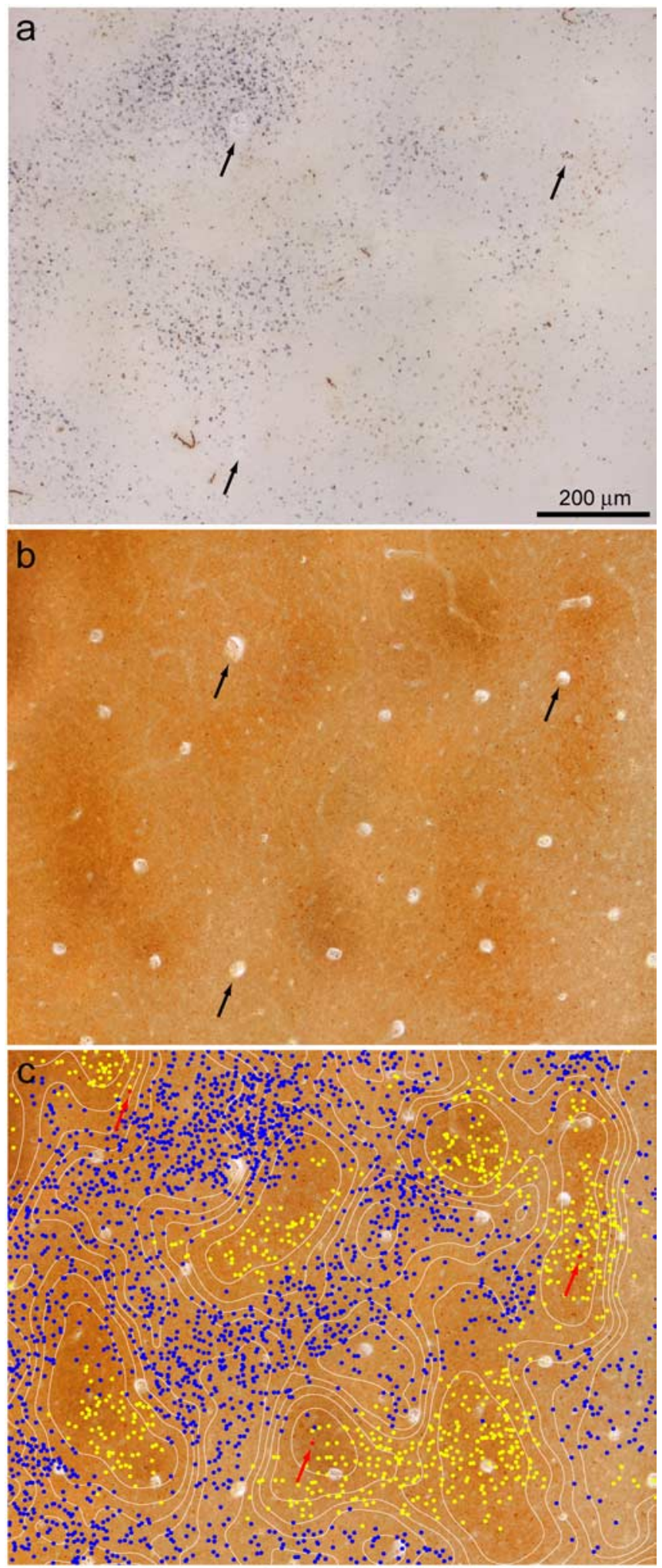

Figure 6. Separate cell population projects to thin stripes and pale stripes. $\boldsymbol{a}$, Boxed region in Figure 5a, showing cells filled with CTB-Au (black) or WGA-HRP (brown). The latter are difficult to see at this magnification. $\boldsymbol{b}$, Adjacent section in layer $2 / 3$ reacted for $\mathrm{C} 0$. Arrows indicate blood vessels used for alignment with the section in $\boldsymbol{a}$. $\boldsymbol{c}$, The CTB-Au-labeled (blue) cells are located in interpatches; the WGA-HRP-filled (yellow) cells are concentrated in patches. There is little intermingling of the two cell populations, and only three cells were double-labeled (red; arrows). The white density contours divide the $\mathrm{C} 0$ activity into six zones of equal area, as in Figure $2 c$.

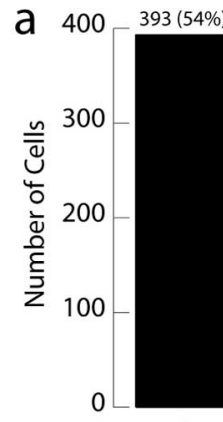

b 600

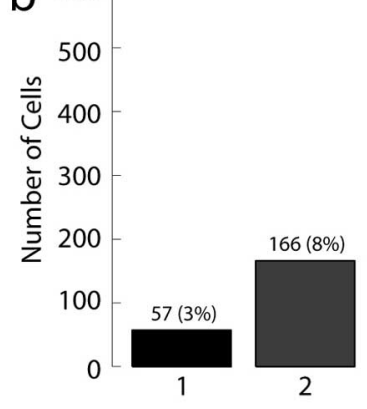

2

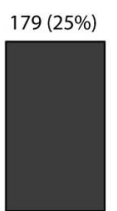

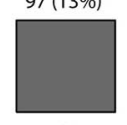

3

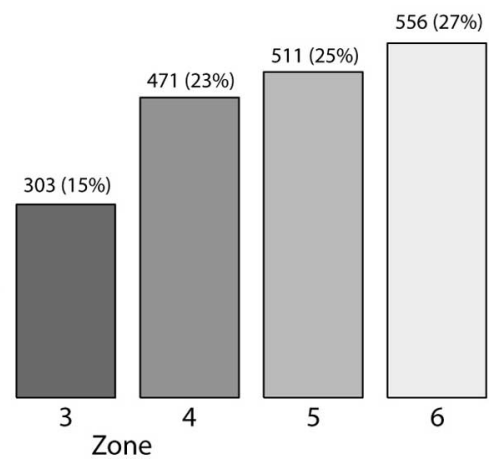

Figure 7. Quantification of projection patterns to thin stripes and pale stripes. $\boldsymbol{a}$, WGA-HRPfilled cells from the thin-stripe injection shown in Figure $5 a$ arise predominately from patches (79\%). $\boldsymbol{b}$, CTB-Au-filled cells from the pale-stripe injection in Figure $5 a$ come mostly from interpatches (89\%).

from V1 is supplied by CO patches. They contain $\sim 80 \%$ of the cells that project to $\mathrm{V} 2$ thin stripes, using a criterion that assigns one-third of the surface area of V1 to the patch compartment. Thus, the density of cells projecting to thin stripes is 8.5 times greater in patches than in interpatches. Moreover, the density of cells innervating thin stripes correlates with the intensity of $\mathrm{CO}$ staining (Fig. 4). This is the best evidence that the V1 projection to V2 thin stripes is predominately from patches, because it does not hinge on exactly what density level is used to define a patch border.

Given that the input to thin stripes arises mostly from patches, is this input segregated from the input to pale stripes? This question was addressed by making paired injections of different retrograde tracers into adjacent pale and thin stripes. The labeling pattern in V1 showed separate, interdigitated fields of CTB-Aufilled cells and WGA-HRP-filled cells, with little overlap (Fig. 6c). Less than one-half a percent of the cells were double-labeled. We conclude that two populations of V1 cells, which are segregated physically, send projections to V2. Patch cells supply thin stripes; interpatch cells feed pale stripes.

Livingstone and Hubel (1984) made nine WGA-HRP injections in two macaques. Two injections hit thin stripes, and each resulted in retrograde filling of cells located in patches. They also made 50 tracer injections in 10 squirrel monkeys. Six landed in thin stripes, and all produced patch labeling. Previously, we reported preferential labeling of patches after 17 of 17 thin-stripe injections (Sincich and Horton, 2002a). Therefore, the conclusion that patches project to thin stripes is supported by 25 of 25 tracer injections.

In these 25 cases, the retrograde label in V1 was measured either by visual inspection (Livingstone and Hubel, 1984) or by thresholding the density of silver grains in retrogradely filled cells (Sincich and Horton, 2002a). Because lightly labeled cells may 
have been overlooked with these approaches, we have now plotted the location of each cell with a light microscope. The same result was obtained, suggesting that our original analysis did not overlook a large population of interpatch cells that fell below threshold because of a scattered distribution or sparse labeling.

The eight cases we selected for quantitative analysis were chosen from the 17 available cases because they featured the most confined examples of injections into V2 thin stripes. Was it valid to analyze only a subset of our cases, choosing those with injections centered within thin stripes that showed minimal extension into surrounding pale stripes? Our goal was to determine the source of input to thin stripes. If an injection lands close to the edge of a thin stripe, the tracer may contaminate an adjacent pale stripe, giving both patch and interpatch label. Therefore, the best strategy for determining the input to thin stripes is to select small, well centered injections. Xiao and Felleman (2004) used the same approach. They reported that 3 of 14 of their tracer injections were clearly restricted to a thin stripe; these three were selected for analysis.

There may be several factors accounting for the difference between our findings and those of Xiao and Felleman (2004). CTB-Au is an extremely efficient tracer, producing thousands of labeled cells in V1 after injection into V2 (supplemental Table 1, available at www.jneurosci.org as supplemental material). Individual sections can also be double-labeled for CTB-Au and CO, so that all the pertinent data are contained within the same tissue section. This eliminates the problem of aligning adjacent sections, which are subject to different degrees of shrinkage during histological processing. When the density of labeled cells per section is comparatively low, one must compensate by analyzing multiple sections. However, relatively few sections are available for plotting the location of cells if separate sections are devoted to CO, Cat-301, FluoroRuby, and biotinylated dextran amine processing. In addition, each section containing labeled cells must be aligned with the $\mathrm{CO}$ section that was used to define the position of the patches. This can be done fairly accurately using blood vessels as a guide, but some error is introduced through this process.

Xiao and Felleman did not unfold the lunate sulcus to reveal the whole pattern of CO stripes in V2. In their material, only a narrow strip of $\mathrm{V} 2, \sim 600 \mu \mathrm{m}$ wide, was visible along the border of the lunate sulcus [Xiao and Felleman (2004), their Fig. $1 B$ ]. It is easier to identify V2 stripes and to define their boundaries when a more complete view of their layout is available. To help distinguish thin stripes from thick stripes, Xiao and Felleman (2004) used Cat-301 immunohistochemistry. Although they did not illustrate their Cat-301 data, this antibody can be useful because it labels thick stripes preferentially (DeYoe et al., 1990).

The most difficult technical aspect of this experiment is to confine one's tracer injection to a given thin stripe. The injection site shown by Xiao and Felleman was illustrated at a different magnification from the adjacent CO-labeled section, and blood vessels were not visible to aid in section alignment [Xiao and Felleman (2004), their Fig. $1 B, C]$. The size and location of a tracer injection relative to the CO-labeled thin stripe provides useful information pertaining to the risk of spillover. Because thin stripes are narrow and flanked by pale stripes, it is easy for tracer spillover to produce inadvertent cell labeling in interpatches. We suspect that tracer spillover was responsible for some of the interpatch labeling in our material. It is telling that our smallest, best-centered CTB-Au injection (supplemental Fig. S7, available at www.jneurosci.org as supplemental material) produced the highest percentage (92\%) of labeled cells in patches.

Xiao and Felleman (2004) assigned a lower percentage of the surface area of V1 to patches, choosing a range of $27-30 \%$. We used a criterion of $33 \%$. A smaller value for the size of a patch will reduce the proportion of labeled cells in patches. We reanalyzed our data and found that $78 \%$ of cells were located in patches using a $30 \%$ area criterion, and $74 \%$ of cells were located in patches using a $27 \%$ area criterion. Thus, our differing definitions of a patch account for only a small portion of the discrepancy in our results.

Our results could be reconciled with those reported by Xiao and Felleman (2004) if thin stripes contain two separate compartments, each receiving a different ratio of patch versus interpatch input from V1. For instance, a color compartment might get a 50:50 patch/interpatch input, whereas another type of compartment might receive a pure patch projection. If the injections we made included both compartments, the result would approximate an 80:20 patch/interpatch distribution of labeled cells. If Xiao and Felleman's injections were smaller and targeted exclusively into color compartments, the result would be a 50:50 patch/interpatch label ratio. This explanation is plausible and not excluded by our data. However, our tracer injections were comparable in size to the color-preferring regions that have been imaged optically in thin stripes (Roe and Ts'o, 1995; Xiao et al., 1999). By chance, some of our injections should have landed in color compartments, producing equal retrograde labeling of patches and interpatches.

In summary, our data uphold the original conclusion of Livingstone and Hubel (1984) that patches project to thin stripes. What is the function of this pathway? Hubel and Livingston (1987) concluded that it represents a specialized color system in the primate. At least 13 other reports have focused on the physiological characteristics of cells in different V2 stripe classes (DeYoe and Van Essen, 1985; Peterhans and von der Heydt, 1993; Levitt et al., 1994; Roe and Ts'o, 1995, 1999; Gegenfurtner et al., 1996; Tamura et al., 1996; Yoshioka and Dow, 1996; Kiper et al., 1997; Xiao et al., 1999, 2003; Ts'o et al., 2001; Moutoussis and Zeki, 2002; Shipp and Zeki, 2002). The verdict from these studies has been mixed, with some investigators confirming that colorselective cells are more prevalent in thin stripes, and others dissenting.

Several optical-imaging reports have provided evidence that color-selective cells are concentrated in thin stripes. However, these studies agree that the match between color regions and thin stripes is imperfect (Roe and Ts'o, 1995; Ts'o et al., 2001; Xiao et al., 2003). Xiao and Felleman (2004) used optical imaging to locate putative color compartments in V2 for their tracer injections. Of 14 targeted injections, three missed thin stripes altogether, and eight straddled the border between a thin stripe and a pale stripe. Only 3 of 14 injections (20\%) were reported to have landed in the center of a thin stripe. This yield was better than we achieved with our injections ( 17 of $187 ; 9 \%$ ) but indicates that it is not easy to target thin stripes by using optical imaging to identify color-selective zones.

It remains uncertain whether thin stripes are enriched in color-coded cells, or whether they contain color modules. In either case, our results indicate that the V1 projection to thin stripes (defined by their appearance in CO-stained sections) arises predominately from patches, not from an equal number of cells in patches and interpatches. Our evidence confirms that different $\mathrm{CO}$ compartments in V1 and V2 are connected in parallel. The existence of these segregated pathways justifies renewed efforts to unravel their functional significance. 


\section{References}

DeYoe EA, Van Essen DC (1985) Segregation of efferent connections and receptive field properties in visual area V2 of the macaque. Nature 317:58-61.

DeYoe EA, Hockfield S, Garren H, Van Essen DC (1990) Antibody labeling of functional subdivisions in visual cortex: Cat-301 immunoreactivity in striate and extrastriate cortex of the macaque monkey. Vis Neurosci 5:67-81.

Farias MF, Gattass R, Piñón MC, Ungerleider LG (1997) Tangential distribution of cytochrome oxidase-rich blobs in the primary visual cortex of macaque monkeys. J Comp Neurol 386:217-228.

Gegenfurtner KR, Kiper DC (2003) Color vision. Annu Rev Neurosci 26:181-206.

Gegenfurtner KR, Kiper DC, Fenstemaker SB (1996) Processing of color, form, and motion in macaque area V2. Vis Neurosci 13:161-172.

Horton JC (1984) Cytochrome oxidase patches: a new cytoarchitectonic feature of monkey visual cortex. Philos Trans R Soc Lond B Biol Sci 304:199-253.

Horton JC, Hubel DH (1981) Regular patchy distribution of cytochrome oxidase staining in primary visual cortex of macaque monkey. Nature 292:762-764.

Hubel DH, Livingstone MS (1987) Segregation of form, color, and stereopsis in primate area 18. J Neurosci 7:3378-3415.

Kiper DC, Fenstemaker SB, Gegenfurtner KR (1997) Chromatic properties of neurons in macaque area V2. Vis Neurosci 14:1061-1072.

LeVay S, Voigt T (1990) Retrograde transneuronal transport of wheat-germ agglutinin to the retina from visual cortex in the cat. Exp Brain Res $82: 77-81$.

Levitt JB, Kiper DC, Movshon JA (1994) Receptive fields and functional architecture of macaque V2. J Neurophysiol 71:2517-2542.

Livingstone MS, Hubel DH (1984) Anatomy and physiology of a color system in the primate visual cortex. J Neurosci 4:309-356.

Livingstone MS, Hubel DH (1988) Segregation of form, color, movement, and depth: anatomy, physiology, and perception. Science 240:740-749.

Llewellyn-Smith IJ, Minson JB, Wright AP, Hodgson AJ (1990) Cholera toxin B-gold, a retrograde tracer that can be used in light and electron microscopic immunocytochemical studies. J Comp Neurol 294:179-191.

Lund JS, Hendrickson AE, Ogren MP, Tobin EA (1981) Anatomical organization of primate visual cortex area VII. J Comp Neurol 202:19-45.

Moutoussis K, Zeki S (2002) Responses of spectrally selective cells in macaque area V2 to wavelengths and colors. J Neurophysiol 87:2104-2112.

Peterhans E, von der Heydt R (1993) Functional organization of area V2 in the alert macaque. Eur J Neurosci 5:509-524.

Purves D, LaMantia A (1993) Development of blobs in the visual cortex of macaques. J Comp Neurol 334:169-175.

Rockland KS, Pandya DN (1979) Laminar origins and terminations of cortical connections of the occipital lobe in the rhesus monkey. Brain Res 179:3-20.

Rockland KS, Virga A (1990) Organization of individual cortical axons projecting from area V1 (area 17) to V2 (area 18) in the macaque monkey. Vis Neurosci 4:11-28.
Roe AW, Ts'o DY (1995) Visual topography in primate V2: multiple representation across functional stripes. J Neurosci 15:3689-3715.

Roe AW, Ts'o DY (1999) Specificity of color connectivity between primate V1 and V2. J Neurophysiol 82:2719-2730.

Rye DB, Saper CB, Wainer BH (1984) Stabilization of the tetramethylbensidine (TMB) reaction product: application for retrograde and anterograde tracing, and combination with immunohistochemistry. J Histochem Cytochem 32:1145-1153.

Shipp S, Zeki S (2002) The functional organization of area V2, I: specialization across stripes and layers. Vis Neurosci 19:187-210.

Sincich LC, Horton JC (2002a) Divided by cytochrome oxidase: a map of the projections from V1 to V2 in macaques. Science 295:1734-1737.

Sincich LC, Horton JC (2002b) Pale cytochrome oxidase stripes in V2 receive the richest projection from macaque striate cortex. J Comp Neurol 447:18-33.

Sincich LC, Horton JC (2003) Independent projection streams from macaque striate cortex to the second visual area and middle temporal area. J Neurosci 23:5684-5692.

Sincich LC, Adams DL, Horton JC (2003) Complete flatmounting of the macaque cerebral cortex. Vis Neurosci 20:663-686.

Tamura H, Sato H, Katsuyama N, Hata Y, Tsumoto T (1996) Less segregated processing of visual information in V2 than in V1 of the monkey visual cortex. Eur J Neurosci 8:300-309.

Tootell RBH, Silverman MS, De Valois RL, Jacobs GH (1983) Functional organization of the second cortical visual area in primates. Science 220:737-739.

Ts'o DY, Roe AW, Gilbert CD (2001) A hierarchy of the functional organization for color, form and disparity in primate visual area V2. Vision Res 41:1333-1349.

Van Essen DC, Newsome WT, Maunsell JH, Bixby JL (1986) The projections from striate cortex (V1) to areas V2 and V3 in the macaque monkey: asymmetries, areal boundaries, and patchy connections. J Comp Neurol 244:451-480.

Weller RE, Kaas JH (1983) Retinotopic patterns of connections of area 17 with visual areas V-II and MT in macaque monkeys. J Comp Neurol 220:253-279.

Wong-Riley M, Carroll EW (1984) Effect of impulse blockage on cytochrome oxidase activity in monkey visual system. Nature 307:262-264.

Wong-Riley MTT (1979) Changes in the visual system of monocularly sutured or enucleated cats demonstrable with cytochrome oxidase histochemistry. Brain Res 171:11-28.

Xiao Y, Felleman DJ (2004) Projections from primary visual cortex to cytochrome oxidase thin stripes and interstripes of macaque visual area 2. Proc Natl Acad Sci USA 101:7147-7151.

Xiao Y, Zych A, Felleman DJ (1999) Segregation and convergence of functionally defined V2 thin stripe and interstripe compartment projections to area V4 of macaques. Cereb Cortex 9:792-804.

Xiao Y, Wang Y, Felleman DJ (2003) A spatially organized representation of colour in macaque cortical area V2. Nature 421:535-539.

Yoshioka T, Dow BM (1996) Color, orientation and cytochrome oxidase reactivity in areas $\mathrm{V} 1, \mathrm{~V} 2$ and $\mathrm{V} 4$ of macaque monkey visual cortex. Behav Brain Res 76:71-88. 